\title{
Stoma Complications: Corticosteroid and Silver Nitrate-Resistant Inflammation Successfully Treated with Topical Tacrolimus
}

\author{
Emanuele Verga ${ }^{a}$ Brinder Chohan ${ }^{b}$ Roberto Verdolini ${ }^{a}$ \\ a Department of Dermatology, and ${ }^{\mathrm{b}}$ Department of Pathology, The Princess Alexandra \\ Hospital NHS Trust, Harlow, UK
}

Dear Editors,

Stomas following colorectal surgery have a significant incidence of complications including chronic inflammation. Potent topical steroids and silver nitrate applications represent the standard treatment, but a considerable number of cases remain unresponsive. We would like to illustrate the case of a-65-year-old female patient who was referred to our Dermatology Department by the Hospital's Stoma Care and Colorectal Services for a persistent severe rash of her ileal stoma, consequent to a procto-colectomy that required 10 years ago for ulcerative colitis. From the time of the operation, despite a number of treatment attempts and a couple of revisions to the ileostomy, the stoma remained inflamed and the patient experienced consistent discomfort with significant impact on her quality of life. Silver Nitrate and several corticosteroid preparations were used topically for years without success. On clinical examination, the stoma presented some slightly raised, friable and oozing plaques and the patient described the area as always being very uncomfortable, painful and sometimes bleeding (Fig. 1a). A patch test excluded a contact dermatitis to any of the components of the stoma pouch material or to other related accessories and cleansing products. Even if carcinoma arising at an ileostomy is a rare occurrence [1], we performed biopsies on the glandular mucosa on the external side of the stoma, which confirmed the absence of any dysplastic change or malignancy. Histology confirmed the presence of florid inflammation and ulceration with fibrino-purulent slough, in keeping with severe ileitis also with ulcerative changes (Fig. 2a, b). Considering the lack of efficacy of the standard treatments, we decided to prescribe tacrolimus $0.1 \%$ ointment to the patient for twice daily application to the stoma, in conjunction with the change of the stoma pouch during the day. The symptoms com- pletely disappeared in around 4 weeks from the beginning of the treatment (Fig. 1b). After more than 1 year, using the same treatment throughout, the stoma was found to be softer and no signs of inflammation were present. The surrounding skin appeared clear. No recurrences or further bleedings have been documented and, more importantly, the pain and discomfort resolved completely and the patient started enjoying a normal life. The most common stoma complications reported in literature are hernia, muco-cutaneous separation, necrosis, retraction, stenosis, prolapse, laceration, infection, abscess and fistula formation [2]. Furthermore, up to $73 \%$ of stoma patients have been reported to experience peristomal skin conditions related to pouching problems. Lastly, granulomas develop often on the stoma, as a result of over-healing of damaged stoma surface, possibly due to friction from the bag, prolonged faecal irritation, irritative or allergic

\section{KARGER}

(C) 2017 S. Karger AG, Basel
Emanuele Verga, MD

Department of Dermatology

The Princess Alexandra Hospital NHS Trust

Hamstel Road, Harlow, Essex CM20 1QX (UK)

E-Mail emanuele.verga @ pah.nhs.uk 

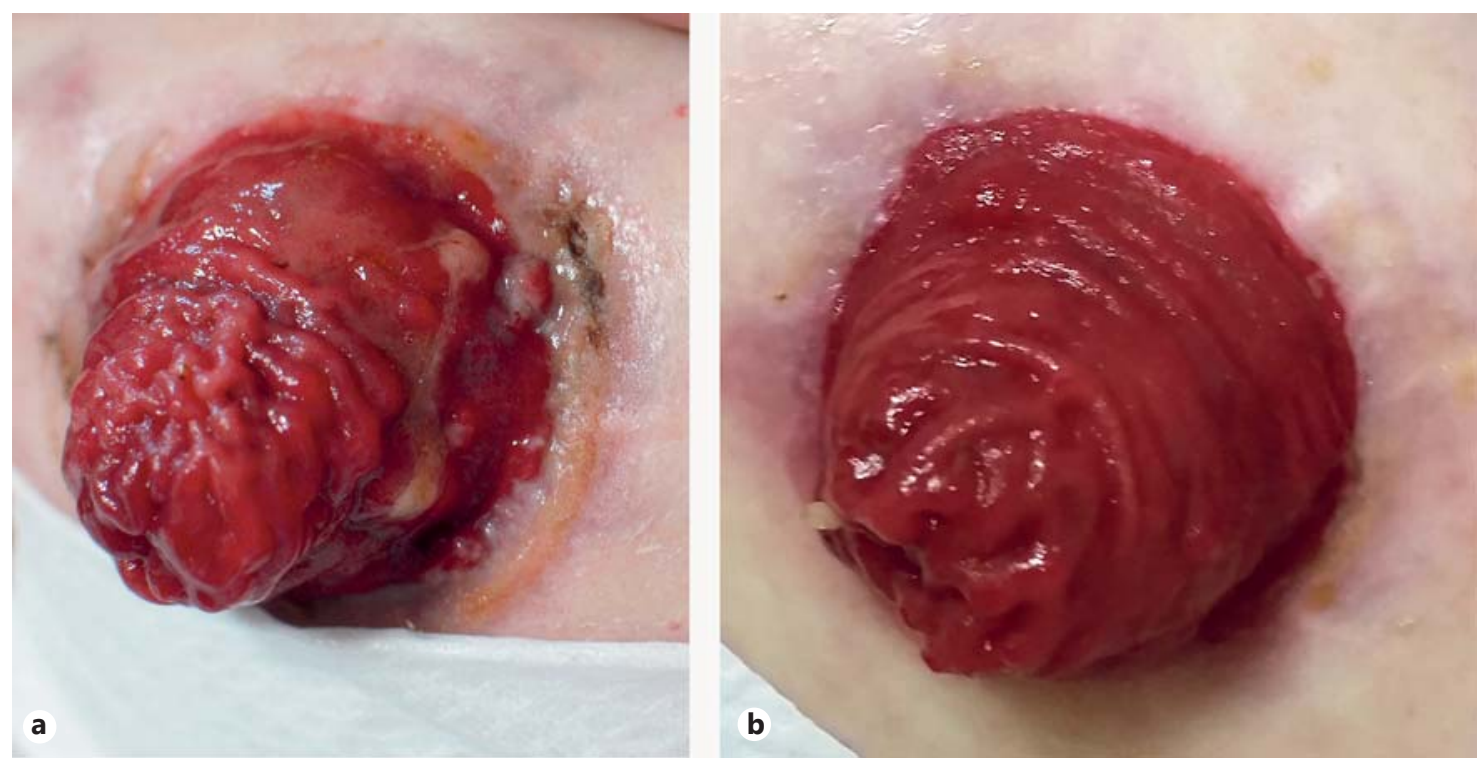

Fig. 1. a Stoma at presentation with inflamed plaques at the base of the stoma present for many years (bleeding was common and the constant pain was heaving a serious impact on the patient's life). b Stoma after 12 weeks of topical treatment with tacrolimus

$0.1 \%$ ointment twice daily. The mucosal surface is clear and the ulcerated nodules covered with fibrin material, visible in the previous picture have disappeared. Discomfort and pain have also resolved.
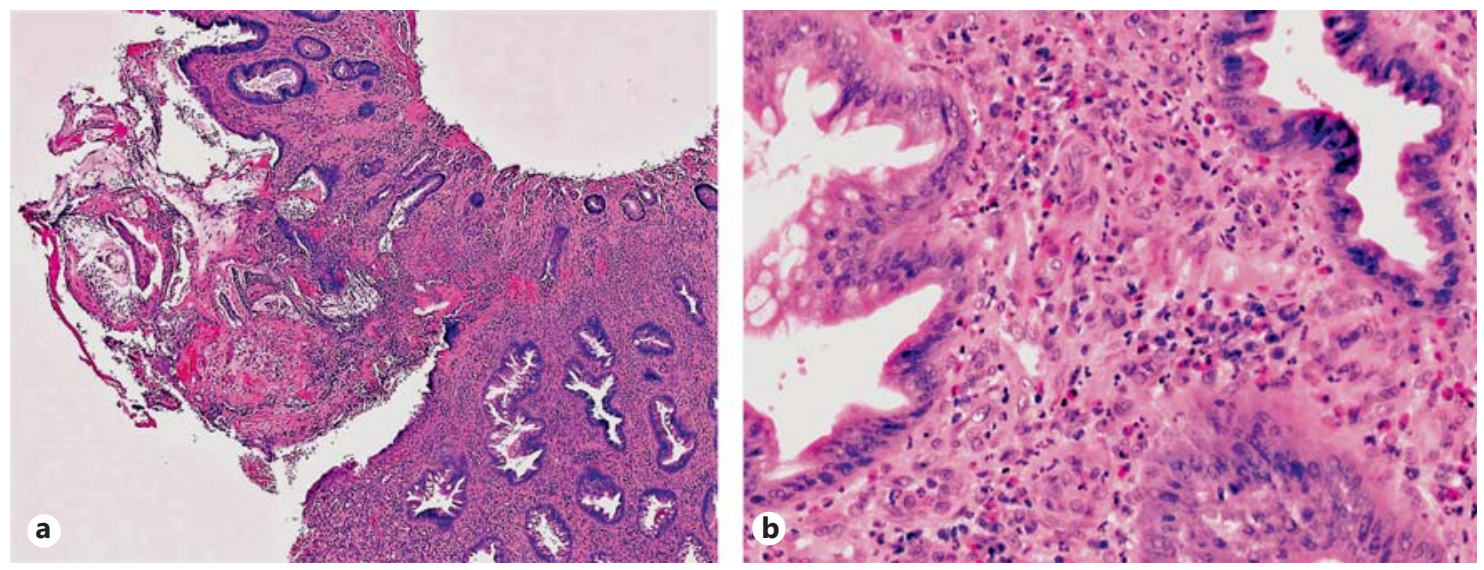

Fig. 2. a Low magnification shows atrophic ileal mucosa, with diffuse inflammation of the submucosal tissue and the presence of focal ulceration $(\mathrm{EE}, \times 20)$. $\mathbf{b}$ Higher magnification demonstrates the presence of both granulocytic and lymphocytic florid infiltrate in the submucosal tissue. Eosinophils are abundant. The overlying epithelium appears atrophic and partially ulcerated $(\mathrm{EE}, \times 40)$. reaction to the pouch, tight clothes over the stoma, causing pain and discomfort $[3,4]$. Tacrolimus is an immunosuppressive macrolide isolated from a Streptomyces that inhibits T-helper lumphocyte (CD-4) activity and Interleukin-2 transcription [5]. In its topical form, it is mainly used for the treatment of atopic eczema but also found to be helpful in other inflammatory conditions driven by T-lymphocytic reaction.
Recent studies conducted on large cohorts of patients confirmed its safety, also for long-term use [6]. Topical tacrolimus has been used on parastomal dermatitis in particular for pyoderma gangrenosum, present in $2 \%$ of inflammatory bowel diseases, such as ulcerative colitis or Crohn's disease [7-9]. We believe that this case may be of interest to colorectal surgeons and health care professionals involved in stoma care as the direct use of topical tacrolimus to successfully treat inflammatory changes of a stoma, to our knowledge, has never been reported before.

\section{Disclosure Statement}

The authors declare no conflicts of interest for this article.
Topical Tacrolimus to Treat Stoma Complications
Dig Surg 2017;34:518-520 DOI: $10.1159 / 000455335$ 


\section{References}

1 Ejtehadi F, Nizamoglu M, Sivakumar R: Squamous cell carcinoma at an ileostomy site-fifty-four years following colectomy for ulcerative colitis: a case report and literature review. Int J Surg Case Rep 2013;4:678-680.

2 Kann BR: Early stomal complications. Clin Colon Rectal Surg 2008;21:23-30.

3 Smith AJ, Lyon CC, Hart CA: Multidisciplinary care of skin problems in stoma patients. Br J Nurs 2002;11:324-330.

4 Nybaek H, Jemec GB: Skin problems in stoma patients. J Eur Acad Dermatol Venereol 2010; 24:249-257.
5 Kino T, Hatanaka H, et al: FK-506, a novel immunosuppressant isolated from a streptomyces. I. Fermentation, isolation, and physico-chemical and biological characteristics. J Antibiot (Tokyo) 1987;40:1249-1255.

6 Siegfried EC, Jaworski JC, Kaiser JD, Hebert AA: Systematic review of published trials: long-term safety of topical corticosteroids and topical calcineurin inhibitors in pediatric patients with atopic dermatitis. BMC Pediatr 2016;16:75.
7 Lyon CC, Stapleton M, Smith AJ, Mendelsohn S, Beck MH, Griffiths CE: Topical tacrolimus in the management of peristomal pyoderma gangrenosum. J Dermatolog Treat 2001;12: 13-17.

8 Altieri M, Vaziri K, Orkin BA: Topical tacrolimus for parastomal pyoderma gangrenosum: a report of two cases. Ostomy Wound Manage 2010;56:56-59.

9 Ruocco E, Sangiuliano S, Gravina AG, et al: Pyoderma gangrenosum: an updated review. J Eur Acad Dermatol Venereol 2009;23:10081017. 